\title{
Ein stereophotogrammetrischer Sensor mit einem Strahlteiler aus Prismen
}

\author{
Christian Keck ${ }^{1}$ Rainer Tutsch ${ }^{1}$ \\ ${ }^{1}$ Institut für Produktionsmesstechnik (IPROM), Technische Universität Braunschweig \\ Schleinitzstraße 20, 38106 Braunschweig
}

\section{Zusammenfassung}

Für die sensorgeführte Mikromontage mit Robotern werden optische Sensoren benötigt, die die räumliche Lage von zu fügenden Bauteilen im laufenden Prozess berührungslos messen. In diesem Beitrag soll ein kompakter stereophotogrammetrischer Sensor für den Einsatz in der Mikromontage vorgestellt werden. Der katadioptrische Sensor arbeitet mit einer Kamera und einem Strahlteiler aus zwei teilverspiegelten Prismen, die fest miteinander verbunden sind. Die Kamera nimmt mit dem Strahlteiler das Messfeld von oben aus zwei unterschiedlichen Raumrichtungen auf. Die beiden Ansichten werden durch den Strahlteiler und das Objektiv getrennt nebeneinander auf den Bildsensor abgebildet, so dass die Aufnahmen mit herkömmlichen stereophotogrammetrischen Verfahren verarbeitet werden können.

Der Strahlteiler kommt ohne bewegliche Elemente aus und verleiht dem Sensor eine besondere Robustheit gegen thermische und mechanische Belastungen. Eine Nut auf der Seite des Strahlteilers, die der Kamera zugewandt ist, begrenzt das Übersprechen zwischen den Ansichten. Mit den Prismen im Strahlteiler werden zusätzliche optische Trennflächen in den Strahlengang zwischen dem Messfeld und der Kamera eingefügt, die bei der Einmessung und dem Betrieb des Sensors besonders beachtet werden müssen.

Keywords: Photogrammetrie, Strahlteiler, Einmessung, Sensor

\section{Einleitung}

Hybride Mikrosysteme werden aus einzelnen Bauelementen gefertigt. Bei der Montage müssen die Bauelemente zusammengesetzt und zum Teil miteinander verbunden werden, zum Beispiel durch Kleben. Für eine erfolgreiche Montage sind Positioniergenauigkeiten in der Größenordnung von $1 \mu \mathrm{m}$ und darunter erforderlich.

Die Montage von Mikrosystemen wird mit Montagerobotern durchgeführt [1]. Die Bauelemente werden zur Montage in Magazinen bereitgestellt. Die Roboter greifen die Bauelemente aus den Magazinen, bringen sie in die Montageposition und halten sie dort, bis der Montageschritt abgeschlossen ist.

Die Genauigkeit des Greifvorgangs liegt weit unter der für die Montage notwendigen Genauigkeit. Während des Abbindens von Klebeverbindungen können sich die Bauteile gegeneinander verdrehen und verschieben. Eine ausreichend genaue Montage ist nur möglich, wenn der Montageprozess mit Sensoren laufend überwacht wird und bei Abweichungen sofort korrigierend eingegriffen wird (sensorgeführte Montage) [1].
Hier soll ein kompakter stereophotogrammetrischer Sensor für die berührungslose Überwachung von Mikromontageprozessen in Echtzeit vorgestellt werden. Der Sensor wird in einem Montageroboter eingesetzt, der im Sonderforschungsbereich SFB 516 entwickelt wurde [1] und ist direkt in die Hand des Roboters integriert, so dass er die Montageszenerie zu jedem Zeitpunkt vollständig überblicken kann. Er erfasst die zu montierenden Bauteile über Kreismarken, die in Planartechnologie auf die Bauteile aufgebracht werden, und misst ihre räumliche Lage.

\section{Aufbau und Funktion des Sensors}

Der katadioptrische Sensor (Schnitt in Abb. 1) nimmt mit einer einzigen Kamera und einem Strahlteiler gleichzeitig eine linke und eine rechte Ansicht des Messfelds auf. Die Kamera ist zusammen mit dem Objektiv und dem Strahlteiler senkrecht über dem Messfeld angeordnet.

Der Strahlteiler (Abb. 2) besteht aus zwei Prismen, deren Seitenflächen verspiegelt sind. Die links und rechts außen angeordneten Spiegel nehmen die linke beziehungsweise die rechte Ansicht auf. Die beiden inneren Spiegel 
bilden die Ansichten durch das Objektiv nebeneinander in die beiden Hälften des Bildsensors der Kamera ab.

Der gefaltete Strahlengang erlaubt es, die Kamera senkrecht über dem Messfeld anzuordnen, so dass ein niedriges Einbauvolumen und eine günstige Masseverteilung erreicht werden können. Herkömmliche stereophotogrammetrische Sensoren benötigen zwei getrennte Kameras, die im Triangulationswinkel gegeneinander verkippt sein müssen, was zu einem wesentlich höheren Platzbedarf und zu einer exzentrischen Masseverteilung führt.

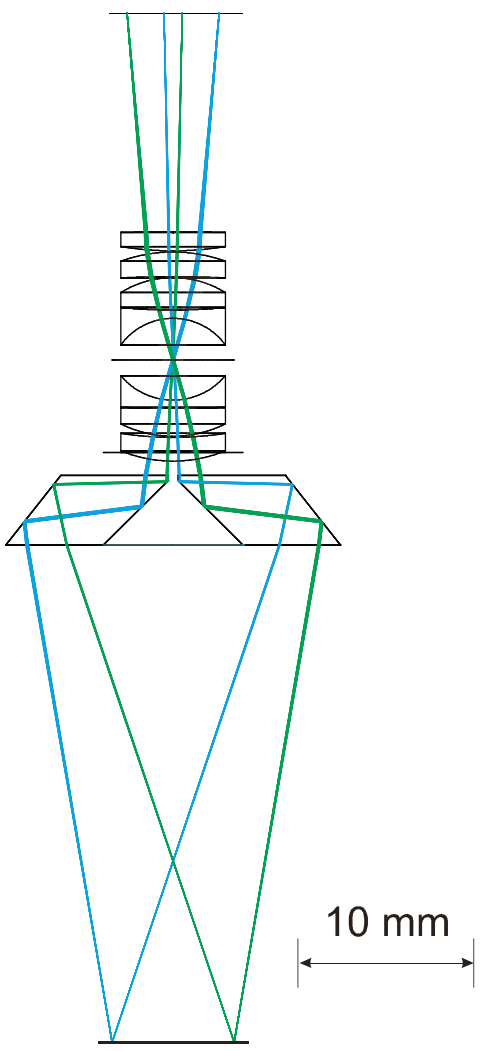

Abb. 1: Ray-Tracing-Simulation des Sensors. Der linke und die rechte Ansicht des Messfelds werden getrennt auf den Bildsensor der Kamera abgebildet.

Die optische Trennung der Ansichten hat den Vorteil, dass herkömmlichen photogrammetrischen Verfahren für die Auswertung der Aufnahmen eingesetzt werden können und keine zusätzlichen Annahmen gemacht werden müssen.

In der Umgebung der vertikalen Mittelachse des Bildsensors kommt es zum Übersprechen zwischen der linken und der rechten Ansicht. Dort blickt die Eintrittspupille der Kamera zugleich auf beide innere Spiegel und nimmt sowohl Teile der linken Ansicht als auch Teile der rechten Ansicht auf. Das Übersprechen ist umso stärker, je größer die Eintrittspupille des
Objektivs und je kleiner die am Objektiv eingestellte Blendenzahl ist.

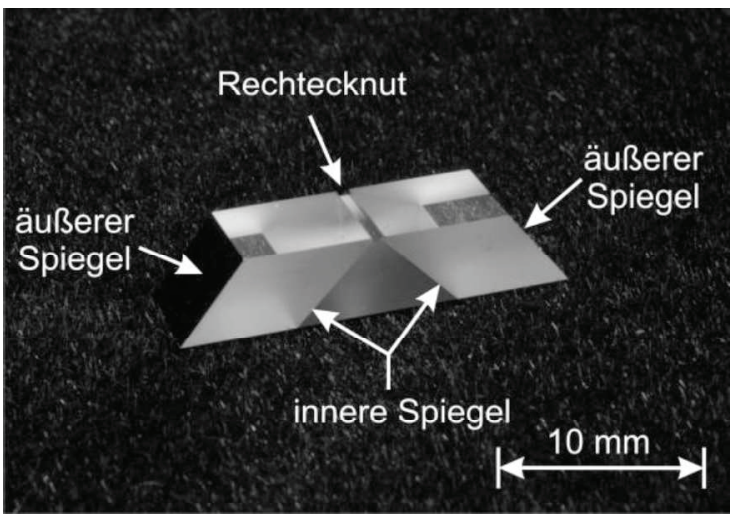

Abb. 2: Strahlteiler. Die beiden äußeren Prismen lenken das Licht aus dem Messfeld in die Kamera. Das mittlere Prisma stellt die mechanische Verbindung zwischen den äußeren Prismen her.

\section{Strahlteiler}

Der Strahlteiler (Abb. 2) besteht aus drei Prismen. Die beiden äußeren spatförmigen Prismen bilden den optisch aktiven Teil. Das dritte $90^{\circ}$-Dreiecksprisma dient lediglich als mechanische Verbindung zwischen den beiden äußeren Prismen.

Um dem Übersprechen zwischen den Ansichten entgegenzuwirken, wurde auf der Kameraseite des Strahlteilers eine Rechtecknut vorgesehen (Abb. 2). Zusätzlich verhindert die Nut Störungen durch Licht, das durch totale interne Reflexion auf der Kameraseite direkt von einem äußeren Prisma in das andere äußere Prisma gelangt.

Die Fertigung des Strahlteilers gestaltet sich einfach. Die Strahlteiler werden aus geschliffenen Glasleisten (Schott N-BK7) hergestellt, die auf einer auf einer ebenen Fläche zusammengeschoben, mit einem UV-Kleber verklebt und in Abschnitte zersägt werden.

Die feste Klebeverbindung zwischen den Prismen macht den Sensor unempfindlich gegen mechanische und thermische Belastungen. Der Strahlteiler kann mit den vorderen und hinteren Seitenflächen an eine ebene Platte geklemmt werden oder mit ihr verklebt werden.

Im Gegensatz zu Sensoren, die mit einem Strahlteiler aus einzelnen, getrennt justierbaren Spiegeln aufgebaut sind [1], kann das Messfeld bei dem hier vorgestellten Sensor nur über den Arbeitsabstand zwischen dem Strahlteiler und der Kamera oder den Auszug der Kamera eingestellt werden. 


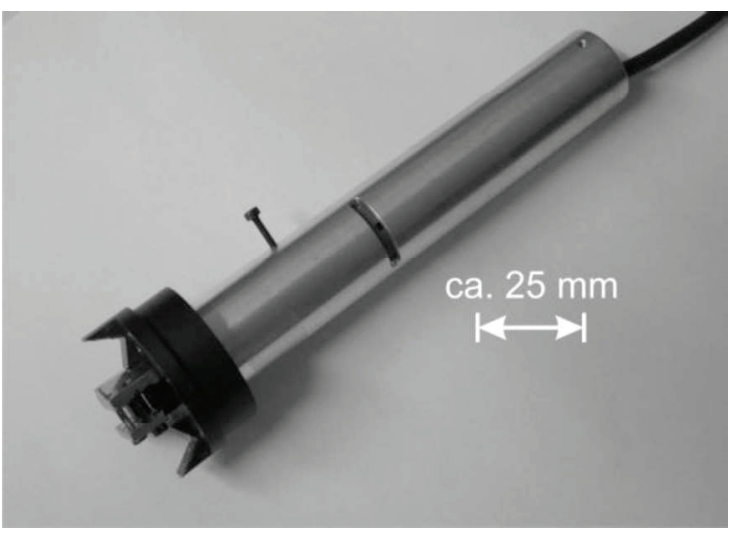

Abb. 3: Montierter Sensor ohne LEDBeleuchtung vor dem Einbau in den Montageroboter.

\section{Prototyp}

Der fertig montierte Sensor (Abb. 3) besteht aus zwei Baugruppen. Die vordere, dem Objekt zugewandte Baugruppe (im Bild links unten, maximaler Außendurchmesser $38 \mathrm{~mm}$, Höhe $25 \mathrm{~mm}$ ) trägt den gegen eine Ebene geklemmten Strahlteiler und die (hier noch nicht montierte) LED-Beleuchtung.

Die hintere, zylindrische Baugruppe (im Bild rechts oben) hält mit ihrem vorderen Teil die CCD-Miniaturkamera. Gleichzeitig dient sie zur Aufnahme des Sensors in die Hand des Montageroboters.

Der freie Arbeitsabstand zwischen dem Sensor und dem Objekt beträgt etwa $30 \mathrm{~mm}$. Das Messfeld ist etwa $6 \mathrm{~mm}$ breit und $10 \mathrm{~mm}$ tief. Der scharf abgebildete Bereich ist etwa $4 \mathrm{~mm}$ hoch. Die Auflösung beträgt etwa $19 \mu \mathrm{m}$ pro Pixel. Der Triangulationswinkel zwischen der linken und der rechten Ansicht beträgt $35^{\circ}$.

\section{Abbildungseigenschaften}

Die Abbildungseigenschaften des Sensors weichen deutlich von den Eigenschaften herkömmlicher stereophotogrammetrischer Sensoren ab.

Eine Probeaufnahme (Abb. 4) eines ebenen, horizontal unter dem Sensor gelagerten Kalibriermusters zeigt, dass nicht die Breite des Bildsensors nicht voll genutzt werden kann. Das vom Sensor erfasste Messfeld erreicht nur ein Seitenverhältnis von etwa 1,8:3, schränkt die Einsatzmöglichkeiten des Sensors in der Mikromontage aber nicht wesentlich ein.

In der Nähe der vertikalen Mittelachse des Bildsensors erscheint das Bild dunkler als in den weiter außen gelegenen Bereichen. Dort begrenzen die inneren Spiegel die aus dem Objekt kommenden Strahlenbündel erheblich, so dass weniger Licht als weiter außen auf den Sensor gelangt.

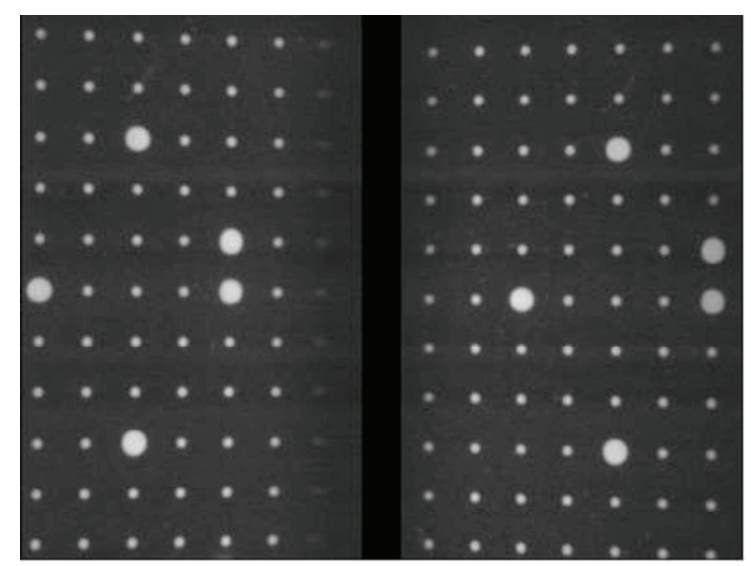

Abb. 4: Aufnahme eines horizontal unter dem Sensor gelagerten ebenen Kalibriermusters. In der Bildmitte begrenzen die inneren Spiegel das in die Kamera gelangende Strahlbündel erheblich und verdunkeln das Bild.

Die dunklere Abbildung an den äußeren Rändern erklärt sich durch die Vignettierung des Objektivs.

Der an den Spiegeln entfaltete Strahlengang (Abb. 5) zeigt die getrennte Abbildung der linken und rechten Ansichten in die beiden Hälften des Bildsensors, wie an den grün gezeichneten Randstrahlen zu sehen ist. Die Kamera wird im entfalteten Strahlengang durch zwei virtuelle Kameras mit jeweils einem Projektionszentrum und einem Bildsensor mit halber Breite ersetzt.

Die aus dem Messfeld kommenden Strahlen werden beim Ein- und Austritt aus den Prismen zweimal gebrochen, bevor sie in die Kamera gelangen. Durch die Brechung an den Eintrittsund Austrittsflächen ist der Winkel zwischen den Mittelachsen der aufgenommenen Ansichten größer als der Winkel zwischen den optischen Achsen der virtuellen Kameras.

In den entfalteten Strahlengang sind auch die vorderen und hinteren Ränder des Bereichs eingetragen, der scharf in die Kamera abgebildet wird. Der scharf abgebildete Bereich wurde aus den Formeln [2] für den scharf abgebildeten Bereich in Luft abgeleitet, während der Verlauf der Randstrahlen bis zum Austritt aus den Prismen durch Strahlverfolgung (Ray Tracing) numerisch berechnet wurde.

Durch die Brechung und die unterschiedlichen optischen Wege durch das Glas auf den Innenund Außenseiten der Prismen sind die Randflächen des scharf abgebildeten Bereichs deutlich zur optischen Achse geneigt. Das Volumen, das durch den Sensor erfasst werden kann, ist kleiner als das Volumen, das mit einem Sensor ohne Prismen erfasst werden kann, bei dem die Strahlen zwischen Messfeld und Kamera nur durch Luft laufen. 
Beim Betrieb des Sensors muss daher besonders darauf geachtet werden, dass die gemessenen Objekte scharf abgebildet werden. Diese Bedingung ist aber bei der Montage der in Planartechnologie gefertigten und niedrigen Mikrobauteile nicht schwierig einzuhalten.

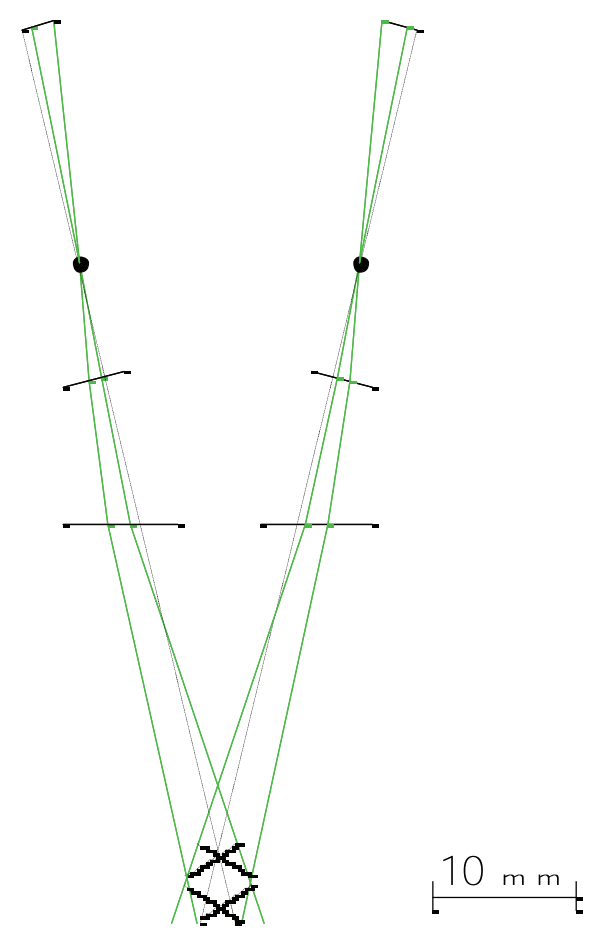

Abb. 5: Entfalteter Strahlengang. Die linke Ansicht wird auf die rechte Hälfte des Bildsensors, die rechte Ansicht auf die linke Hälfte des Bildsensors abgebildet.

\section{Erste Versuche}

Die Genauigkeit eines photogrammetrischen Sensors wird wesentlich durch seine messtechnische Stabilität sowie durch die Genauigkeit bestimmt, mit der der Sensor eingemessen wird. Voraussetzung für eine genaue Einmessung ist ein Modell, das die auf dem Weg vom Objekt zum Bildsensor entstehenden Verzeichnungen ausreichend genau beschreibt.

Erste Versuche, den Sensor mit einem herkömmlichen photogrammetrischen Kameramodell [2], das radialsymmetrische und tangentiale Verzeichnungen des Objektivs sowie die "elektronischen" Verzeichnungen des Bildsensors durch die Rechteckform der Sensorpixel und den Abtastvorgang berücksichtigt, führten zu Längenmessabweichungen von bis zu $10 \mu \mathrm{m}$, die auf den ersten Blick niedrig erscheinen, aber für den Einsatz des Sensors in der Mikromontage nicht ausreichen.
Als Beispiel soll hier die Messung (Abb. 6) an einem Mikrobauteil in der Mitte des Messfelds angeführt werden. Dabei wurde der Abstand zwischen zwei Kreismarken mit einem Durchmesser von $150 \mu \mathrm{m}$ und einem Nennabstand von $2 \mathrm{~mm}$ in 200 Wiederholungen gemessen.

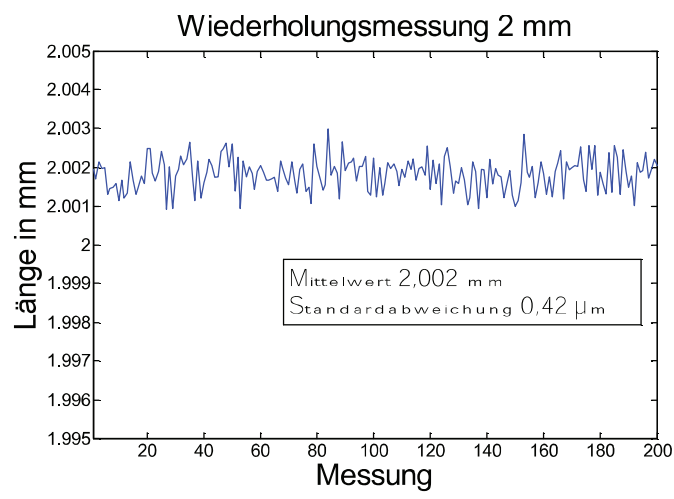

Abb. 6: Messung des räumlichen Abstands zwischen zwei Kreismarken in der Mitte des Messvolumens in 200 Wiederholungen. Der Abstand wird $2 \mu \mathrm{m}$ zu groß gemessen.

Die Wiederholpräzision von 0,4 $\mu \mathrm{m}$ zeigt, dass der Sensor ausreichend stabil ist, um eine photogrammetrische Kalibrierung erfolgreich durchführen zu können. Die systematische Messabweichung beträgt etwa $2 \mu \mathrm{m}$ und zeigt, dass das für die Einmessung verwendete Modell nicht ausreichend genau ist.

\section{Photogrammetrische Einmessung}

Bei dem hier beschriebenen Sensor müssen neben den Verzeichnungen des Objektivs auch die durch die Brechung an den Prismen verursachten Verzeichnungen berücksichtigt werden.

Bringt man eine ebene Glasscheibe in den Strahlengang zwischen einem Objekt und einer Kamera ein, so dass die Eintritts- und Austrittsebenen senkrecht zur optischen Achse der Kamera ausgerichtet sind, können die entstehenden Verzeichnungen durch einen veränderten Abbildungsmaßstab (beziehungsweise eine vergrößerte Kammerkonstante) und zusätzliche radialsymmetrische Verzeichnungen beschrieben werden [2][3].

Der entfaltete Strahlengang des Sensors (Abb. 5) zeigt, dass die Eintrittsflächen der Prismen zur optischen Achse der Kamera geneigt sind, während die Austrittsflächen senkrecht zur optischen Achse stehen. Die Brechung an den Prismen verursacht daher Verzeichnungen, die nicht mehr alleine durch einen anderen Abbildungsmaßstab und radialsymmetrische Verzeichnungen beschrieben werden können. Bei der oben erwähnten Einmessung wurden vermutlich der geänderte Maßstab und die 
radialsymmetrischen Verzeichnungen gut erfasst, während der Einfluss der geneigten Eintrittsflächen nicht zutreffend modelliert wurde.

Verzeichnungen durch zusätzliche optische Trennflächen werden schon seit langer Zeit untersucht, zum Beispiel im Zusammenhang mit der Photogrammetrie von Objekten unter Wasser. Kotowski [3] hat 1987 einen allgemeinen Ansatz beschrieben, mit dem optische Trennflächen beim Bündelblockausgleich durch eine rechnerische Nachverfolgung der Strahlbündel vom Objekt zum Sensor zusätzlich berücksichtigt werden können. Voraussetzung ist, dass die Grundform der Trennflächen durch ein parametrisiertes mathematisches Modell beschrieben werden kann. Die Trennflächen können eine feste Orientierung zum Objekt haben, zum Beispiel eine glatten Wasseroberfläche über einem Objekt, oder eine feste Orientierung zur Kamera haben, wie zum Beispiel eine Schutzscheibe für eine gekapselte Kamera.

Im Vergleich zu den komplexen Szenarien, die mit dem Verfahren von Kotowski beschrieben werden können, gestaltet sich das bei der Einmessung der Kamera auftretende Problem einfach. Die Prismen sind fest mit der Kamera verbunden. Die Wellenlänge der grünen LEDBeleuchtung ist gut bekannt. Ebenso ist die Brechzahl des für die Prismen verwendeten Glases gut bekannt und unter den Betriebsbedingungen des Sensors sehr stabil.

Hier soll auf eine frühere Idee von Girndt [4] zurückgegriffen werden, mit der die Wirkung einer einzelnen ebenen Trennfläche berücksichtigt werden kann. Girndt beschreibt ein Verfahren, bei dem nach einer geeignet gewählten Koordinatentransformation der Eintrittspunkt in die Trennfläche aus dem Objektpunkt und dem Projektionszentrum der Kamera direkt berechnet werden kann. Der Eintrittspunkt wird auf den Bildpunkt in der Kamera abgebildet.

Hier wird das Verfahren in geänderter Form auf Systeme mit zwei optischen Trennflächen angewendet werden, indem der zu einem Objektpunkt gehörige Bildpunkt durch numerische Optimierung iterativ bestimmt wird. Dieses Modell soll in eine neue objektorientierte Software zum Bündelblockausgleich integriert werden, die beliebige Varianten für Kameramodelle unterstützt.

\section{Zusammenfassung}

Der vorliegende Beitrag stellt einen katadioptrischen Stereosensor für die Mikromontage vor. Der Sensor arbeitet mit einer einzelnen Kamera und einem Strahlteiler aus teilweise verspiegelten und miteinander verklebten Prismen, der dem Sensor eine hohe messtechnische Stabilität verleihen soll.

Der Strahlteiler nimmt die linke und die rechte Ansicht des Messfelds getrennt auf und bildet sie nebeneinander in die Kamera ab. Der Einsatz des Strahlteilers macht es möglich, die Kamera unmittelbar über dem Messvolumen anzuordnen und einen Sensor zu bauen, der den besonderen Anforderungen beim Einsatz in einem Roboter für die Mikromontage gerecht wird.

Das Abbildungsverhalten des Sensors wird stark durch die Brechung der aus dem Messfeld kommenden Lichtstrahlen an den ebenen Ein- und Austrittsflächen der Prismen bestimmt. Das Abbildungsverhalten muss bei der Einmessung des Sensors und im Betrieb besonders beachtet werden.

Der Sensor zeigt bei der Messung von Abständen zwischen planaren Kreismarken eine hohe Wiederholpräzision. Dies ist eine gute Voraussetzung für eine erfolgreiche genaue Einmessung. Für die Einmessung das ein Modell verwendet werden, das sich an dem Vorschlag von Girndt orientieren soll.

\section{Danksagung}

Die Autoren danken der Braunschweig International Graduate School of Metrology (IGSM) für die Unterstützung der Arbeiten.

\section{Literaturnachweis}

[1] C. Keck, M. Berndt, R. Tutsch: Stereophotogrammetry in Microasssembly. In. S. Büttgenbach, A. Burisch, J. Hesselbach (Herausgeber): Design and Manufacturing of Active Microsystems. Springer-Verlag, Berlin und Heidelberg 2011. Seiten $309-326$.

[2] N. Luhmann: Nahbereichsphotogrammetrie. Grundlagen, Methoden und Anwendungen. 3. Auflage. Wichmann-Verlag, Berlin 2010.

[3] R. Kotowski: Zur Berücksichtigung lichtbrechender Flächen im Strahlenbündel. Deutsche Geodätische Kommission. Reihe C (Dissertationen), Heft 330. Verlag der Bayerischen Akademie der Wissenschaften, München 1987.

[4] U. Girndt: Analytische Behandlung von einigen Grundaufgaben der Zweimedien-Photogrammetrie. Deutsche Geodätische Kommission. Reihe C (Dissertationen), Heft 196. Verlag der Bayerischen Akademie der Wissenschaften, München 1973. 